\title{
The Mitochondrial Translocator Protein and Arrhythmogenesis in Ischemic Heart Disease
}

\author{
Lukas J. Motloch, Jun Hu, and Fadi G. Akar \\ Cardiovascular Institute, Icahn School of Medicine at Mount Sinai, Hess Center for Science \& Medicine, \\ One Gustave L. Levy Place, P.O. Box 1030, New York, NY 1002, USA
}

Correspondence should be addressed to Fadi G. Akar; fadi.akar@mssm.edu

Received 8 January 2015; Accepted 18 March 2015

Academic Editor: Silvana Hrelia

Copyright (c) 2015 Lukas J. Motloch et al. This is an open access article distributed under the Creative Commons Attribution License, which permits unrestricted use, distribution, and reproduction in any medium, provided the original work is properly cited.

\begin{abstract}
Mitochondrial dysfunction is a hallmark of multiple cardiovascular disorders, including ischemic heart disease. Although mitochondria are well recognized for their role in energy production and cell death, mechanisms by which they control excitationcontraction coupling, excitability, and arrhythmias are less clear. The translocator protein (TSPO) is an outer mitochondrial membrane protein that is expressed in multiple organ systems. The abundant expression of TSPO in macrophages has been leveraged to image the immune response of the heart to inflammatory processes. More recently, the recognition of TSPO as a regulator of energy-dissipating mitochondrial pathways has extended its utility from a diagnostic marker of inflammation to a therapeutic target influencing diverse pathophysiological processes. Here, we provide an overview of the emerging role of TSPO in ischemic heart disease. We highlight the importance of TSPO in the regenerative process of reactive oxygen species (ROS) induced ROS release through its effects on the inner membrane anion channel (IMAC) and the permeability transition pore (PTP). We discuss evidence implicating TSPO in arrhythmogenesis in the settings of acute ischemia-reperfusion injury and myocardial infarction.
\end{abstract}

\section{Introduction}

The translocator protein (TSPO), formerly known as the peripheral benzodiazepine receptor (PBR), is an $18 \mathrm{kDa}$ mitochondrial protein consisting of 169 amino acids [1]. Arranged in five transmembrane domains, TSPO is a nuclearencoded protein localized on chromosome 22q13.31 [2-4]. TSPO, which exhibits a highly conserved structure, has been cloned from multiple species including man, dog, cow, pig, rat, and mouse $[2,5]$. Enriched in the outer mitochondrial membrane (OMM), TSPO is an integral component of a macromolecular complex of proteins that regulates cell survival and death pathways $[1,2]$. Found in most organs within the body, TSPO exhibits robust expression in secretory and glandular tissue, kidney, liver, brain, and heart [1, 2, 6]. The widespread distribution of TSPO is consistent with its diverse physiological functions. These include, but are not limited to, membrane biogenesis, heme biosynthesis, immunomodulation, bioenergetics, redox balance, apoptosis, and cholesterol binding and transport [1, 7-11]. As such, altered TSPO expression and activity in the heart may have important implications for a wide spectrum of cardiovascular disorders. In this review, we highlight the link between TSPO and the pathological process of reactive oxygen species (ROS) induced ROS release (RIRR) which we propose to be a master regulator of electrical dysfunction on one hand and cell death on the other hand. We implicate TSPO in the adverse remodeling associated with ischemia-reperfusion injury and myocardial infarction, both of which are major risk factors for arrhythmias (Figure 1).

\section{TSPO as a Mediator of ROS Induced ROS Release}

Mitochondria synthesize adenine triphosphate (ATP) through oxidative phosphorylation. This highly regulated process is fueled by the mitochondrial membrane potential $\left(\Delta \Psi_{m}\right)$, which forms the proton-motive force used to shuttle electrons across the electron transport chain [12-14]. In 

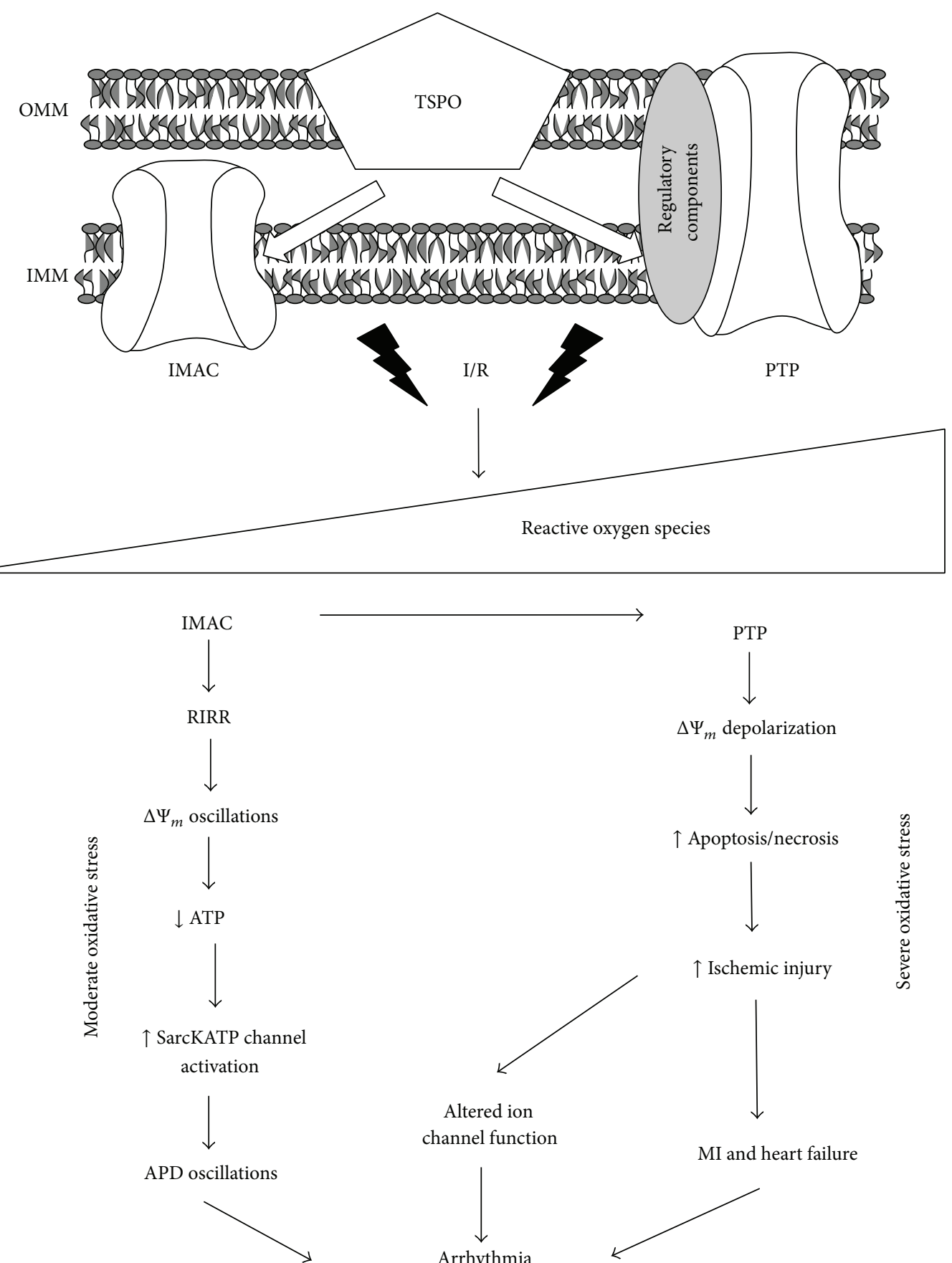

$\uparrow$ Apoptosis/necrosis

Altered ion channel function
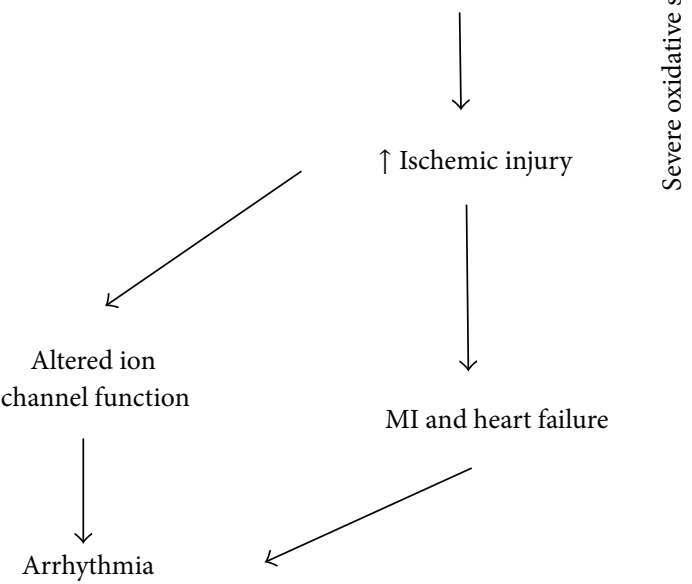

FIGURE 1: Schematic illustration of the central role of TSPO in the pathogenesis of arrhythmias in response to oxidative stress. APD: action potential duration; IMM: inner mitochondrial membrane; OMM: outer mitochondrial membrane; RIRR: ROS induced ROS release.

addition to ATP, ROS are also generated when electrons that leak from the ETC combine with oxygen to form superoxide anions $\left(\mathrm{O}_{2}^{-}\right)$[15]. In healthy myocardium, ROS production is countered by efficient antioxidant defense systems which maintain physiological redox balance. In diseased myocardium, overproduction and/or defective scavenging of ROS often leads to oxidative stress (OS). Understanding mechanisms by which myocardial ROS levels are amplified to cause OS is critical for our ability to combat prevalent diseases, in which OS is a hallmark feature.
The concept of RIRR was born from studies by Zorov et al. $[16,17]$ and Aon et al. [18-20]. These investigators demonstrated that local ROS injury within a discrete region of a cardiomyocyte can rapidly accumulate across a critical mass of the mitochondrial network to cause cellular OS. As such, RIRR describes a fundamental mechanism by which cardiac mitochondria react to elevated ROS levels by stimulating endogenous ROS production. Indeed, this regenerative, autocatalytic process ultimately results in cellular dysfunction and death [17]. Distinct modes of RIRR have been postulated 
based on their dependence on either the mitochondrial permeability transition pore (PTP) or the inner membrane anion channel (IMAC) $[21,22]$, both of which are modulated by TSPO. As will be highlighted below, TSPO-mediated regulation of RIRR can influence postischemic arrhythmias directly via IMAC and indirectly via PTP.

\section{TSPO Regulates RIRR via IMAC}

Multiple ROS-sensitive ion channels exist in the mitochondrial membrane. Of key importance is the IMAC which was discovered in liver and heart mitochondria [23]. In a series of seminal studies, Garlid and colleagues demonstrated the anion selectivity of IMAC and its dependence on $\mathrm{pH}$ and temperature [23-27]. As with other anion channels, IMAC was readily inhibited by stilbene-22' -disulfonates [28]. IMAC activity was also reduced by $\mathrm{Ca}^{2+}, \mathrm{Mg}^{2+}[23,25-$ $27,29]$, and a variety of cationic amphiphilic agents [23, 24]. Studies of drug-channel interactions were consistent with the notion that IMAC mediated the efflux of $\mathrm{O}_{2}^{-}[30,31]$. This, in turn, implicated the channel in the regulation of cellular bioenergetics and redox properties [32]. Although the molecular structure of IMAC remained elusive, its tight regulation by TSPO-acting ligands ( $4^{\prime} \mathrm{ClDzpm}, \mathrm{PK} 11195$, and IX protoporphyrin) suggested a close interaction between its pore-forming subunit in the inner mitochondrial membrane (IMM) and the regulatory protein TSPO in the OMM [12, 24, 33]. Despite these studies, the nonspecific effects of many TSPO ligands on calcium handling and other cellular processes such as contractility and excitability have confounded our ability to pinpoint the direct role of TSPO per se in cardiac pathophysiology [34-38]. Nonetheless, studies by O'Rourke and colleagues have elegantly documented the role of IMAC in RIRR-mediated metabolic and electrophysiological instabilities. Indeed, these investigators demonstrated that photo-induced oxidation of a discrete region within the cardiac myocyte can unleash a regenerative process of ETC-derived ROS that was dependent on IMAC. Once ROS levels across a critical portion (60-70\%) of the mitochondrial network exceeded a given threshold, sustained $\Delta \Psi_{m}$ oscillations were initiated. This highly nonlinear property was coined as "mitochondrial criticality" $[20,39]$. Using computational modelling, Cortassa and colleagues demonstrated that the frequency of the synchronized cell-wide mitochondrial oscillations was strongly modulated by ROS scavengers and the rate of oxidative phosphorylation [40]. Importantly, RIRR-evoked mitochondrial oscillations gave rise to cellular electrophysiological oscillations that were dependent on the cyclical activation of sarcolemmal $\mathrm{K}_{\mathrm{ATP}}$ channels. Activation of these normally dormant channels is thought to be protective as they act to preserve energy at a time of increased metabolic demand. However, increasing $\mathrm{K}$ efflux through these channels can induce rapid and heterogeneous action potential duration (APD) shortening and suppress myocyte excitability in a manner that predisposes to reentrant arrhythmias $[12,13,39,41]$. Importantly, both metabolic and electrophysiological oscillations could be readily abolished by TSPO ligands, which functionally reduce cardiomyocyte ROS levels [18-20, 39, 42].
More recently, we extended the concept of RIRR from a subcellular phenomenon to one occurring at the organ level. Using optical mapping approaches, we demonstrated the functional significance of RIRR in terms of arrhythmia propensity [42-45]. In a model of moderate OS produced by relatively brief challenge with $\mathrm{H}_{2} \mathrm{O}_{2}$ perfusion, TSPO inhibition abolished the large amplitude secondary $\mathrm{O}_{2}{ }^{-}$ peak which arose following, not during, the exogenous oxidative challenge [43]. Prevention of this secondary $\mathrm{O}_{2}{ }^{-}$ peak abrogated ventricular fibrillation and suppressed the frequency of arrhythmogenic triggers [43]. Indeed, these findings highlighted the importance of IMAC-mediated $\mathrm{O}_{2}{ }^{-}$ release as a driving force for RIRR associated arrhythmias in intact myocardium.

\section{TSPO May Regulate RIRR via PTP}

In addition to IMAC, the PTP is also activated in response to rising ROS levels $[21,46]$. However, a hierarchal pattern seems to govern the activation sequence of the two channels [18]: IMAC is activated firstly in response to moderate levels of OS followed by the activation of the nonspecific, high-conductance PTP. Despite considerable debate over its molecular composition, the PTP is strongly regulated by the voltage-dependent anion channel (VDAC) in the OMM, the adenine nucleotide translocase (ANT) in the IMM, and Cyclophilin D in the mitochondrial matrix [46]. Importantly, the Bernardi group has recently demonstrated the role of dimers of the ATP synthase in the formation of the PTP [47]. A causative relationship between OS-induced mitochondrial permeability transition and $\Delta \Psi_{m}$ depolarization has also been demonstrated in numerous studies. Zorov et al. [16, 17] found a direct correlation between PTP activation and myocyte death. These findings, combined with evidence that $\Delta \Psi_{m}$ depolarization was abolished by the Cyclophilin D inhibitor Cyclosporin A (CsA), suggested an important role for PTPmediated $\Delta \Psi_{m}$ depolarization in RIRR-mediated apoptosis [16].

We recently investigated the efficacy of the PTP desensitizer CsA in protecting against OS-induced mitochondrial and electrical dysfunction at the intact heart level [48]. Unlike our previous study [43], we chose a severe model of continuous $\mathrm{H}_{2} \mathrm{O}_{2}$ challenge which reliably and predictably caused irreversible $\Delta \Psi_{m}$ depolarization within a 30 min timeframe, likely through PTP activation [48]. Our experiments uncovered a dual role for CsA in either protecting or impairing cardiac function depending on the cellular milieu [48]. Specifically, we found that CsA-mediated cardioprotection in this severe model of oxidative challenge required mitochondrial $\mathrm{K}_{\mathrm{ATP}}\left(\mathrm{mK}_{\mathrm{ATP}}\right)$ channel activation through a protein kinase $C$ dependent pathway [48]. Increasing $\mathrm{mK}_{\mathrm{ATP}}$ activity during CsA administration was required for limiting OSinduced electromechanical dysfunction. On the other hand, CsA administration during conditions that prevented $\mathrm{mK}_{\text {ATP }}$ channel activation exerted unintended proarrhythmic consequences possibly through accelerated APD shortening [48]. Our findings addressed existing controversy in the basic and clinical literature surrounding the utility of CsA as a cardioprotective agent [48-53]. 
In addition to Cyclophilin D, there is some evidence that the PTP may also be regulated by TSPO through interaction with the VDAC and ANT [54-56]. This area, however, requires further clarification considering the elegant findings of Šileikyte et al. who recently discounted a major role of TSPO in the regulation of the PTP by the outer membrane [57]. As such, more studies aimed at defining the macromolecular complex of proteins that forms the PTP are critical to our ability to identify new therapeutic targets. This is especially important considering the apparent shortcomings of CsA, which fails to abrogate laser flash-induced $\Delta \Psi_{m}$ oscillations [19] and promotes rather than prevents arrhythmias during conditions that prohibit $\mathrm{mK}_{\mathrm{ATP}}$ channel activation [48]. Indeed, novel approaches aimed at limiting ROS-mediated PTP opening are likely to have major clinical implications. As such, targeting regulatory PTP components via TSPO may have a dual beneficial impact by improving electrical function while simultaneously promoting cell survival in response to OS $[54,55]$. Using the TSPO ligands SSR180575 and $4^{\prime}$ ClDzp, Leducq et al. successfully inhibited $\Delta \Psi_{m}$ depolarization in isolated cardiac mitochondria after excessive ROS exposure. In addition, both agents reduced apoptosis linked events, including cytochrome c release, caspase- 3 activation, and DNA fragmentation [58].

\section{Role of TSPO in Postischemic Arrhythmias}

Ischemic heart disease is a major public health epidemic and a leading cause of morbidity and mortality worldwide $[59,60]$. Ischemic injury predisposes to myocardial infarction, heart failure, arrhythmias, and sudden cardiac death. Prompt restoration of oxygenated blood flow to the ischemic myocardium is required for limiting the extent of irreversible cell damage and death [61]. Unfortunately, restoration of blood flow, in itself, results in additional cardiac damage known as reperfusion injury [62]. Such ROS-mediated damage is more severe when reperfusion therapy is delayed. Reperfusion-mediated redox imbalance and cytosolic calcium overload promote mechanoelectrical dysfunction and the genesis of lethal arrhythmias shortly upon reperfusion.

Although earlier studies showed that TSPO-acting ligands which reduced ROS levels were effective in abolishing metabolic and electrophysiological oscillations, their impact on arrhythmias in clinically relevant scenarios was not established until more recently [42]. We investigated whether protection against mitochondrial depolarization could translate into an antiarrhythmic benefit in response to ischemia-reperfusion injury. IMAC blockade blunted ischemia-induced APD shortening and the onset of inexcitability in a dose-dependent manner [42]. In contrast, IMAC activation using FGIN-1-27 accelerated APD shortening and resulted in an early form of conduction failure during ischemia [42]. Specifically, hearts that underwent IMAC activation prior to ischemia exhibited heightened sensitivity to ischemia. Using high-resolution optical mapping, we identified discrete areas of conduction block in these hearts during early ischemia that persisted upon reperfusion and likely promoted the formation of reentrant activity underlying postischemic ventricular fibrillation [42]. Remarkably, IMAC blockade, which stabilizes $\Delta \Psi_{m}$ in vitro, suppressed these arrhythmias and promoted the rapid recovery of the action potential upon reperfusion [42]. The protective effect of IMAC blockade on postischemic electrical function was also evident in a rabbit model of ischemia-reperfusion injury [35]. Of note, the antiarrhythmic effect afforded by TSPO ligands was not evident in hearts treated with the PTP desensitizer, CsA. This reinforces IMAC as a primary mitochondrial mediator of acute postischemic arrhythmias.

\section{Role of TSPO in Myocardial Infarction}

Myocardial infarction (MI) is a global health epidemic that predisposes to heart failure and arrhythmias [60]. Indeed, the majority of cardiac-related deaths occur in patients who develop MI as a consequence of coronary artery disease [63]. In those patients the risk of cardiac arrest is approximately 4-6 times that of the general population [64]. PTP opening in response to severe ischemia-reperfusion injury results in $\mathrm{MI}$ as a consequence of myocyte loss to necrosis/apoptosis [46]. Since MI is a major risk factor for heart failure and arrhythmias, PTP inhibition may exert an indirect antiarrhythmic effect by reducing infarct size and improving overall cardiac function [65]. In a small placebo-controlled trial involving 58 STEMI (ST segment elevation myocardial infarction) patients, CsA administration was associated with smaller infarcts at the time of reperfusion [66]. Despite these encouraging clinical findings, the efficacy of CsA in preventing arrhythmias remained unclear $[49,51,53]$. In fact, several experimental, preclinical [52], and clinical findings [50] have cast serious doubts regarding the overall utility and safety profile of CsA. Hence, new approaches for combatting MI are urgently needed.

TSPO ligands inhibit PTP function through a distinct mechanism of action that is not dependent on Cyclophilin $\mathrm{D}[54,56,67]$. As such, these agents may be an exciting alternative to CsA. Indeed, SSR180575 improved left ventricular function and reduced infarct size in rat and rabbit models of ischemia-reperfusion injury [58]. Moreover, TSPO inhibition with $4^{\prime} \mathrm{ClDzp}$ improved the rate of oxidative phosphorylation, inhibited PTP opening, and limited the rate of apoptosis in isolated mitochondria [67]. Interestingly, the cardioprotective efficacies of $4^{\prime} \mathrm{ClDzp}$ and PK11195 were similar to those elicited by ischemic preconditioning or diazoxide treatment [67]. Similar results were obtained by Xiao et al. [68] who observed increased ETC activity and reduced ROS levels. These favorable bioenergetic properties were associated with improved recovery of left ventricular mechanical function and a diminished rate of arrhythmias upon reperfusion [68]. Initial results in large animals were also encouraging. Specifically, treatment of pigs with $4^{\prime} \mathrm{ClDzp}$ at the time of reperfusion following 60 minutes of left anterior descending coronary artery occlusion was associated with rapid ST segment resolution and a trend towards reduced infarct size in the absence of major hemodynamic complications [69]. 
Recently, the effects of a new TSPO ligand TRO40303 which binds at the cholesterol site of the mitochondrial protein were examined. As with $4^{\prime} \mathrm{ClDZP}, \mathrm{TRO} 40303$ inhibited PTP opening and apoptosis in response to simulated ischemia-reperfusion or hydrogen peroxide challenge in adult or neonatal cardiomyocytes, respectively [70]. These cellular effects correlated with reduced ROS levels and infarct size in anesthetized rats [70]. The efficacy of TRO40303 even when delivered as a single bolus injection before reperfusion provided the impetus needed for testing in humans. In a Phase I double-blind placebo-controlled clinical trial involving 72 volunteers [71], TRO40303 met the safety criteria. This naturally paved the way for a Phase II trial [71, 72]. MITOCARE is an ongoing multicenter, randomized, doubleblind, placebo-controlled survey evaluating the safety and efficacy of TRO40303 for the reduction of reperfusion injury in patients undergoing percutaneous coronary intervention for acute MI [72].

Although TSPO-mediated PTP inhibition may be of major therapeutic value in the future, this strategy is complicated by several issues. Of note, the use of higher concentrations of TSPO ligands (i.e., $>100 \mathrm{uM} 4^{\prime} \mathrm{ClDZP}$ and $>40 \mathrm{uM}$ PK11195) can elicit cardiotoxic effects such as mitochondrial swelling $[67,73,74]$. This highly undesirable property, which is consistent with PTP activation rather than inhibition, raises important questions regarding the nature of the regulation of the PTP by TSPO. On one hand, TSPO-mediated IMAC activation induces $\mathrm{O}_{2}{ }^{-}$outflow from the mitochondrial matrix. If this outflow is large enough, it stimulates the regenerative process of RIRR that culminates in mitochondrial depolarization and PTP opening. On the other hand, low levels of IMAC activity may mediate a protective mechanism that limits the build-up of mitochondrial ROS levels required for PTP opening. As such, complete IMAC inhibition using high doses of TSPO ligands may paradoxically accelerate rather than prevent PTP formation. Determining whether the cytotoxic effects observed using high concentrations of these ligands are a consequence of natural cross talk between the PTP and IMAC or due to nonspecific effects of these agents will be of major importance going forward. Finally, TSPO can regulate PTP opening independently of RIRR. By modulating mitochondrial cholesterol uptake and the subsequent generation of oxyterols [75], TSPO ligands can exert additional cardioprotective effects. Taken together, these studies implicate PTP inhibition in the beneficial (namely, infarct sparing) effects of TSPO-acting ligands that reduce ROS levels. This important property is expected to significantly reduce the propensity for arrhythmias by reducing the size of the infarct and slowing the progression towards heart failure.

\section{Summary and Future Directions}

Mounting evidence implicates TSPO in OS-related dysfunction, including arrhythmogenesis. Of key importance is the putative ability of TSPO to modulate two prominent energy dissipating channels, namely, IMAC and the PTP. In doing so, TSPO ligands have shown considerable promise in combatting postischemic ventricular fibrillation (via IMAC inhibition) and MI (via PTP inhibition).

Despite the apparent success of TSPO antagonists in multiple experimental settings, major concerns exist. Of note, high concentrations of TSPO ligands can paradoxically activate the PTP in isolated mitochondria $[67,73,74]$, either by disrupting the cross talk between the PTP and IMAC or by eliciting nonspecific effects. Indeed, there is substantial evidence that these agents affect sarcolemmal ion channels, such as the L-type calcium current, even at baseline $[35,38$, 76-78]. This, in turn, alters excitation-contraction coupling and contractility. Moreover, findings that $4^{\prime} \mathrm{ClDzp}$ results in decreased APD in rabbit [35], unchanged APD in guinea pig [42], and prolonged APD in rat (our own unpublished observations) are consistent with an effect on the transient outward $\mathrm{K}$ current. Indeed, species-dependent changes in cardiac inotropy and chronotropy by available TSPO ligands further confound the widespread translation of these agents to the clinic [34-38]. Finally, the wide distribution of TSPO receptors in the body presents an added challenge for pharmacological approaches that would likely exert numerous extracardiac effects.

Considering the limitations of currently available TSPO ligands, new biological approaches for modulating TSPO expression and activity in the heart are needed. One such approach is the use of cardiac-specific adeno-associated viral vector serotypes carrying silencing RNAs against TSPO. Indeed, the future design and implantation of such gene therapy approaches may revolutionize the treatment of a host of common cardiovascular disorders in which mitochondrial dysfunction is a hallmark mechanism. In the meantime, a more systematic understanding of the role of TSPO in cardiovascular health and disease is needed.

\section{Abbreviations}

APD: Action potential duration

ANT: Adenine nucleotide translocase

ATP: Adenine triphosphate

CsA: Cyclosporin A

DIDS: Stilbene-22'-disulfonates

ETC: Electron transport chain

IMAC: Inner membrane anion channel

IMM: Inner mitochondrial membrane

OMM: Outer mitochondrial membrane

OS: Oxidative stress

PBR: Peripheral benzodiazepine receptor

PTP: Permeability transition pore

RIRR: ROS induced ROS release

TSPO: Translocator protein

VDAC: Voltage-dependent anion channel.

\section{Conflict of Interests}

The authors declare that there is no conflict of interests regarding the publication of this paper. 


\section{References}

[1] V. Papadopoulos, M. Baraldi, T. R. Guilarte et al., “Translocator protein $(18 \mathrm{kDa})$ : new nomenclature for the peripheral-type benzodiazepine receptor based on its structure and molecular function," Trends in Pharmacological Sciences, vol. 27, no. 8, pp. 402-409, 2006.

[2] A. Batarseh and V. Papadopoulos, "Regulation of translocator protein $18 \mathrm{kDa}$ (TSPO) expression in health and disease states," Molecular and Cellular Endocrinology, vol. 327, no. 1-2, pp. 1-12, 2010.

[3] Y. J. Chang, R. T. McCabe, H. Rennert et al., "The human 'peripheral-type' benzodiazepine receptor: regional mapping of the gene and characterization of the receptor expressed from cDNA," DNA and Cell Biology, vol. 11, no. 6, pp. 471-480, 1992.

[4] J. Riond, M. G. Mattei, M. Kaghad et al., "Molecular cloning and chromosomal localization of a human peripheral-type benzodiazepine receptor," European Journal of Biochemistry, vol. 195, no. 2, pp. 305-311, 1991.

[5] L. Veenman and M. Gavish, "The peripheral-type benzodiazepine receptor and the cardiovascular system. Implications for drug development," Pharmacology and Therapeutics, vol. 110, no. 3, pp. 503-524, 2006.

[6] C. Giatzakis and V. Papadopoulos, "Differential utilization of the promoter of peripheral-type benzodiazepine receptor by steroidogenic versus nonsteroidogenic cell lines and the role of Sp1 and Sp3 in the regulation of basal activity," Endocrinology, vol. 145, no. 3, pp. 1113-1123, 2004.

[7] J.-P. Faure, H. Baumert, Z. Han et al., "Evidence for a protective role of trimetazidine during cold ischemia: targeting inflammation and nephron mass," Biochemical Pharmacology, vol. 66, no. 11, pp. 2241-2250, 2003.

[8] S. Taketani, H. Kohno, M. Okuda, T. Furukawa, and R. Tokunaga, "Induction of peripheral-type benzodiazepine receptors during differentiation of mouse erythroleukemia cells. A possible involvement of these receptors in heme biosynthesis," Journal of Biological Chemistry, vol. 269, no. 10, pp. 7527-7531, 1994.

[9] S. R. Torres, T. S. Fröde, G. M. Nardi et al., "Anti-inflammatory effects of peripheral benzodiazepine receptor ligands in two mouse models of inflammation," European Journal of Pharmacology, vol. 408, no. 2, pp. 199-211, 2000.

[10] A. A. Yeliseev and S. Kaplan, "TspO of Rhodobacter sphaeroides. A structural and functional model for the mammalian peripheral benzodiazepine receptor," The Journal of Biological Chemistry, vol. 275, no. 8, pp. 5657-5667, 2000.

[11] J.-J. Lacapère and V. Papadopoulos, "Peripheral-type benzodiazepine receptor: structure and function of a cholesterolbinding protein in steroid and bile acid biosynthesis," Steroids, vol. 68 , no. 7-8, pp. 569-585, 2003.

[12] F. G. Akar, "Mitochondrial targets for arrhythmia suppression: is there a role for pharmacological intervention?" Journal of Interventional Cardiac Electrophysiology, vol. 37, no. 3, pp. 249258, 2013.

[13] D. A. Brown and B. O'Rourke, "Cardiac mitochondria and arrhythmias," Cardiovascular Research, vol. 88, no. 2, pp. 241249, 2010.

[14] V. Adam-Vizi, "Production of reactive oxygen species in brain mitochondria: contribution by electron transport chain and non-electron transport chain sources," Antioxidants \& Redox Signaling, vol. 7, no. 9-10, pp. 1140-1149, 2005.
[15] J. F. Turrens, "Mitochondrial formation of reactive oxygen species," Journal of Physiology, vol. 552, no. 2, pp. 335-344, 2003.

[16] D. B. Zorov, C. R. Filburn, L.-O. Klotz, J. L. Zweier, and S. J. Sollott, "Reactive oxygen species (ROS)-induced ROS release: a new phenomenon accompanying induction of the mitochondrial permeability transition in cardiac myocytes," Journal of Experimental Medicine, vol. 192, no. 7, pp. 1001-1014, 2000.

[17] D. B. Zorov, M. Juhaszova, and S. J. Sollott, "Mitochondrial ROS-induced ROS release: an update and review," Biochimica et Biophysica Acta-Bioenergetics, vol. 1757, no. 5-6, pp. 509-517, 2006.

[18] M. A. Aon, S. Cortassa, C. Maack, and B. O'Rourke, "Sequential opening of mitochondrial ion channels as a function of glutathione redox thiol status," The Journal of Biological Chemistry, vol. 282, no. 30, pp. 21889-21900, 2007.

[19] M. A. Aon, S. Cortassa, E. Marbán, and B. O’Rourke, “Synchronized whole cell oscillations in mitochondrial metabolism triggered by a local release of reactive oxygen species in cardiac myocytes," The Journal of Biological Chemistry, vol. 278, no. 45, pp. 44735-44744, 2003.

[20] M. A. Aon, S. Cortassa, and B. O’Rourke, “The fundamental organization of cardiac mitochondria as a network of coupled oscillators," Biophysical Journal, vol. 91, no. 11, pp. 4317-4327, 2006.

[21] N. R. Brady, A. Hamacher-Brady, H. V. Westerhoff, and R. A. Gottlieb, "A wave of reactive oxygen species (ROS)-induced ROS release in a sea of excitable mitochondria," Antioxidants \& Redox Signaling, vol. 8, no. 9-10, pp. 1651-1665, 2006.

[22] L. Yang, P. Korge, J. N. Weiss, and Z. Qu, "Mitochondrial oscillations and waves in cardiac myocytes: insights from computational models," Biophysical Journal, vol. 98, no. 8, pp. 1428-1438, 2010.

[23] K. D. Garlid and A. D. Beavis, "Evidence for the existence of an inner membrane anion channel in mitochondria," Biochimica et Biophysica Acta, vol. 853, no. 3-4, pp. 187-204, 1986.

[24] A. D. Beavis, "On the inhibition of the mitochondrial inner membrane anion uniporter by cationic amphiphiles and other drugs," The Journal of Biological Chemistry, vol. 264, no. 3, pp. 1508-1515, 1989.

[25] A. D. Beavis, "Properties of the inner membrane anion channel in intact mitochondria," Journal of Bioenergetics and Biomembranes, vol. 24, no. 1, pp. 77-90, 1992.

[26] A. D. Beavis and K. D. Garlid, "The mitochondrial inner membrane anion channel. Regulation by divalent cations and protons," The Journal of Biological Chemistry, vol. 262, no. 31, pp. 15085-15093, 1987.

[27] A. D. Beavis and M. Powers, "Temperature dependence of the mitochondrial inner membrane anion channel: the relationship between temperature and inhibition by magnesium," The Journal of Biological Chemistry, vol. 279, no. 6, pp. 4045-4050, 2004.

[28] A. D. Beavis and H. Davatol-Hag, "The mitochondrial inner membrane anion channel is inhibited by DIDS," Journal of Bioenergetics and Biomembranes, vol. 28, no. 2, pp. 207-214, 1996.

[29] A. D. Beavis and M. F. Powers, "On the regulation of the mitochondrial inner membrane anion channel by magnesium and protons," The Journal of Biological Chemistry, vol. 264, no. 29, pp. 17148-17155, 1989.

[30] A. Paky, J. R. Michael, T. M. Burke-Wolin, M. S. Wolin, and G. H. Gurtner, "Endogenous production of superoxide by rabbit 
lungs: effects of hypoxia or metabolic inhibitors," Journal of Applied Physiology, vol. 74, no. 6, pp. 2868-2874, 1993.

[31] M.-A. Takahashi and K. Asada, "Superoxide anion permeability of phospholipid membranes and chloroplast thylakoids," Archives of Biochemistry and Biophysics, vol. 226, no. 2, pp. 558$566,1983$.

[32] T. L. Vanden Hoek, L. B. Becker, Z. Shao, C. Li, and P. T. Schumacker, "Reactive oxygen species released from mitochondria during brief hypoxia induce preconditioning in cardiomyocytes," The Journal of Biological Chemistry, vol. 273, no. 29, pp. 18092-18098, 1998.

[33] K. W. Kinnally, D. B. Zorov, Y. N. Antonenko, S. H. Snyder, M. W. Mcenery, and H. Tedeschi, "Mitochondrial benzodiazepine receptor linked to inner membrane ion channels by nanomolar actions of ligands," Proceedings of the National Academy of Sciences of the United States of America, vol. 90, no. 4, pp. 13741378, 1993.

[34] G. T. Bolger, S. Abraham, N. Oz, and B. A. Weissman, "Interactions between peripheral-type benzodiazepine receptor ligands and an activator of voltage-operated calcium channels," Canadian Journal of Physiology and Pharmacology, vol. 68, no. 1, pp. 40-45, 1990.

[35] D. A. Brown, M. A. Aon, F. G. Akar, T. Liu, N. Sorarrain, and B. O'Rourke, "Effects of 4 ' -chlorodiazepam on cellular excitationcontraction coupling and ischaemia-reperfusion injury in rabbit heart," Cardiovascular Research, vol. 79, no. 1, pp. 141-149, 2008.

[36] Y. Edoute, J. Giris, S. A. Ben-Haim et al., "Ro 5-4864 and PK 11195, but not diazepam, depress cardiac function in an isolated working rat heart model," Pharmacology, vol. 46, no. 4, pp. 224230, 1993.

[37] R. S. Leeuwin, A. Zeegers, J. van Hamme, and H. van Wilgenburg, "Modification of cardiac actions of RO 05-4864 by PK 11195 and flumazenil in the perfused rat heart," Life Sciences, vol. 61, no. 17, pp. 1631-1642, 1997.

[38] B. A. Weissman, G. T. Bolger, and P. K. Chiang, "Interactions between nitrogen oxide-containing compounds and peripheral benzodiazepine receptors," FEBS Letters, vol. 260, no. 2, pp. 169$172,1990$.

[39] M. A. Aon, S. Cortassa, F. G. Akar, D. A. Brown, L. Zhou, and B. O'Rourke, "From mitochondrial dynamics to arrhythmias," International Journal of Biochemistry and Cell Biology, vol. 41, no. 10, pp. 1940-1948, 2009.

[40] S. Cortassa, M. A. Aon, R. L. Winslow, and B. O’Rourke, “A mitochondrial oscillator dependent on reactive oxygen species," Biophysical Journal, vol. 87, no. 3, pp. 2060-2073, 2004.

[41] B. O’Rourke, B. M. Ramza, and E. Marban, "Oscillations of membrane current and excitability driven by metabolic oscillations in heart cells," Science, vol. 265, no. 5174, pp. 962966, 1994.

[42] F. G. Akar, M. A. Aon, G. F. Tomaselli, and B. O'Rourke, “The mitochondrial origin of postischemic arrhythmias," The Journal of Clinical Investigation, vol. 115, no. 12, pp. 3527-3535, 2005.

[43] N. Biary, C. Xie, J. Kauffman, and F. G. Akar, "Biophysical properties and functional consequences of reactive oxygen species (ROS)-induced ROS release in intact myocardium," Journal of Physiology, vol. 589, no. 21, pp. 5167-5179, 2011.

[44] A. R. Lyon, P. J. Joudrey, D. Jin et al., "Optical imaging of mitochondrial function uncovers actively propagating waves of mitochondrial membrane potential collapse across intact heart," Journal of Molecular and Cellular Cardiology, vol. 49, no. 4, pp. 565-575, 2010.
[45] C. Xie, N. Biary, C. G. Tocchetti et al., "Glutathione oxidation unmasks proarrhythmic vulnerability of chronically hyperglycemic guinea pigs," The American Journal of PhysiologyHeart and Circulatory Physiology, vol. 304, no. 7, pp. H916H926, 2013.

[46] C. Penna, M.-G. Perrelli, and P. Pagliaro, "Mitochondrial pathways, permeability transition pore, and redox signaling in cardioprotection: Therapeutic implications," Antioxidants and Redox Signaling, vol. 18, no. 5, pp. 556-599, 2013.

[47] V. Giorgio, S. von Stockum, M. Antoniel et al., "Dimers of mitochondrial ATP synthase form the permeability transition pore," Proceedings of the National Academy of Sciences of the United States of America, vol. 110, no. 15, pp. 5887-5892, 2013.

[48] C. Xie, J. Kauffman, and F. G. Akar, "Functional crosstalk between the mitochondrial PTP and KATP channels determine arrhythmic vulnerability to oxidative stress," Frontiers in Physiology, vol. 5, article 264, 2014.

[49] D. Arteaga, A. Odor, R. M. López et al., "Impairment by cyclosporin A of reperfusion-induced arrhythmias," Life Sciences, vol. 51, no. 14, pp. 1127-1134, 1992.

[50] S. Ghaffari, B. Kazemi, M. Toluey, and N. Sepehrvand, "The effect of prethrombolytic cyclosporine-A injection on clinical outcome of acute anterior ST-elevation myocardial infarction," Cardiovascular Therapeutics, vol. 31, no. 4, pp. e34-e39, 2013.

[51] W.-J. Ko, F.-L. Lin, S.-S. Wang, and S.-H. Chu, "Hypomagnesia and arrhythmia corrected by replacing cyclosporine with FK506 in a heart transplant recipient," The Journal of Heart and Lung Transplantation, vol. 16, no. 9, pp. 980-982, 1997.

[52] R. H. Lie, N. Stoettrup, E. Sloth, J. M. Hasenkam, R. Kroyer, and T. T. Nielsen, "Post-conditioning with cyclosporine A fails to reduce the infarct size in an in vivo porcine model," Acta Anaesthesiologica Scandinavica, vol. 54, no. 7, pp. 804-813, 2010.

[53] K. D. Schreiner, K. Kelemen, J. Zehelein et al., "Biventricular hypertrophy in dogs with chronic AV block: effects of cyclosporin A on morphology and electrophysiology," The American Journal of Physiology-Heart and Circulatory Physiology, vol. 287, no. 6, pp. H2891-H2898, 2004.

[54] J. Sileikyte, V. Petronilli, A. Zulian et al., "Regulation of the inner membrane mitochondrial permeability transition by the outer membrane translocator protein (peripheral benzodiazepine receptor)," The Journal of Biological Chemistry, vol. 286, no. 2, pp. 1046-1053, 2011.

[55] A. Zulian, J. Šileikyte, V. Petronilli et al., “The translocator protein (peripheral benzodiazepine receptor) mediates ratselective activation of the mitochondrial permeability transition by norbormide," Biochimica et Biophysica Acta: Bioenergetics, vol. 1807, no. 12, pp. 1600-1605, 2011.

[56] M. W. Mcenery, A. M. Snowman, R. R. Trifiletti, and S. H. Snyder, "Isolation of the mitochondrial benzodiazepine receptor: association with the voltage-dependent anion channel and the adenine nucleotide carrier," Proceedings of the National Academy of Sciences of the United States of America, vol. 89, no. 8, pp. 3170-3174, 1992.

[57] J. Šileikyte, E. Blachly-Dyson, R. Sewell et al., "Regulation of the mitochondrial permeability transition pore by the outer membrane does not involve the peripheral benzodiazepine receptor (Translocator Protein of $18 \mathrm{kDa}$ (TSPO))," The Journal of Biological Chemistry, vol. 289, no. 20, pp. 13769-13781, 2014.

[58] N. Leducq, F. Bono, T. Sulpice et al., "Role of peripheral benzodiazepine receptors in mitochondrial, cellular, and cardiac damage induced by oxidative stress and ischemia-reperfusion," 
Journal of Pharmacology and Experimental Therapeutics, vol. 306, no. 3, pp. 828-837, 2003.

[59] World Health Organization, Facts and Figures: The World Health Report, World Health Organization, Geneva, Switzerland, 2003.

[60] D. Lloyd-Jones, R. J. Adams, T. M. Brown et al., "Executive summary: heart disease and stroke statistics-2010 update: a report from the American Heart Association," Circulation, vol. 121, no. 7, pp. 948-954, 2010.

[61] P. T. O’Gara, F. G. Kushner, D. D. Ascheim et al., "2013 ACCF/AHA guideline for the management of ST-elevation myocardial infarction: executive summary: a report of the American College of Cardiology Foundation/American Heart Association Task Force on practice guidelines," Circulation, vol. 127, no. 4, pp. 529-555, 2013.

[62] P. Pagliaro, F. Moro, F. Tullio, M.-G. Perrelli, and C. Penna, "Cardioprotective pathways during reperfusion: focus on redox signaling and other modalities of cell signaling," Antioxidants and Redox Signaling, vol. 14, no. 5, pp. 833-850, 2011.

[63] D. J. Lerner and W. B. Kannel, "Patterns of coronary heart disease morbidity and mortality in the sexes: a 26 -year followup of the Framingham population," American Heart Journal, vol. 111, no. 2, pp. 383-390, 1986.

[64] L. A. Cupples, D. R. Gagnon, and W. B. Kannel, "Long- and short-term risk of sudden coronary death," Circulation, vol. 85, no. 1, supplement, pp. I11-I18, 1992.

[65] E. J. Griffiths and A. P. Halestrap, "Mitochondrial non-specific pores remain closed during cardiac ischaemia, but open upon reperfusion," Biochemical Journal, vol. 307, no. 1, pp. 93-98, 1995.

[66] C. Piot, P. Croisille, P. Staat et al., "Effect of cyclosporine on reperfusion injury in acute myocardial infarction," The New England Journal of Medicine, vol. 359, no. 5, pp. 473-481, 2008.

[67] F. N. Obame, R. Zini, R. Souktani, A. Berdeaux, and D. Morin, "Peripheral benzodiazepine receptor-induced myocardial protection is mediated by inhibition of mitochondrial membrane permeabilization," The Journal of Pharmacology and Experimental Therapeutics, vol. 323, no. 1, pp. 336-345, 2007.

[68] J. Xiao, D. Liang, H. Zhang, Y. Liu, F. Li, and Y.-H. Chen, " 4 ' -chlorodiazepam, a translocator protein $(18 \mathrm{kDa})$ antagonist, improves cardiac functional recovery during postischemia reperfusion in rats," Experimental Biology and Medicine, vol. 235, no. 4, pp. 478-486, 2010.

[69] J. Terrovitis, L. Katsaros, M. Tsamatsoulis et al., "Cardioprotection by stimulation of mitochondrial benzodiazepine receptors during reperfusion, in a porcine acute ischemia-reperfusion model," Journal of the American College of Cardiology, vol. 59, no. 13, supplement, p. E545, 2012.

[70] S. Schaller, S. Paradis, G. A. Ngoh et al., "TRO40303, a new cardioprotective compound, inhibits mitochondrial permeability transition," Journal of Pharmacology and Experimental Therapeutics, vol. 333, no. 3, pp. 696-706, 2010.

[71] S. Le Lamer, S. Paradis, H. Rahmouni et al., "Translation of TRO40303 from myocardial infarction models to demonstration of safety and tolerance in a randomized Phase I trial," Journal of Translational Medicine, vol. 12, no. 1, article 38, 2014.

[72] D. Atar, J.-L. Abitbol, H. Arheden et al., "Rationale and design of the 'MITOCARE' study: a phase II, multicenter, randomized, double-blind, placebo-controlled study to assess the safety and efficacy of TRO40303 for the reduction of reperfusion injury in patients undergoing percutaneous coronary intervention for acute myocardial infarction," Cardiology, vol. 123, no. 4, pp. 201207, 2012.
[73] B. Chelli, A. Falleni, F. Salvetti, V. Gremigni, A. Lucacchini, and C. Martini, "Peripheral-type benzodiazepine receptor ligands: mitochondrial permeability transition induction in rat cardiac tissue," Biochemical Pharmacology, vol. 61, no. 6, pp. 695-705, 2001.

[74] J. Li, J. Wang, and Y. Zeng, "Peripheral benzodiazepine receptor ligand, PK11195 induces mitochondria cytochrome c release and dissipation of mitochondria potential via induction of mitochondria permeability transition," European Journal of Pharmacology, vol. 560, no. 2-3, pp. 117-122, 2007.

[75] S. Paradis, V. Leoni, C. Caccia, A. Berdeaux, and D. Morin, "Cardioprotection by the TSPO ligand $4^{\prime}$-chlorodiazepam is associated with inhibition of mitochondrial accumulation of cholesterol at reperfusion," Cardiovascular Research, vol. 98, no. 3, pp. 420-427, 2013.

[76] M. Holck and W. Osterrieder, "The peripheral high affinity benzodiazepine binding site is not coupled to the cardiac $\mathrm{Ca}^{2+}$ channel," European Journal of Pharmacology, vol. 118, no. 3, pp. 293-301, 1985.

[77] G. Le Fur, M. Mestre, T. Carriot et al., "Pharmacology of peripheral type benzodiazepine receptors in the heart," Progress in Clinical and Biological Research, vol. 192, pp. 175-186, 1985.

[78] M. Mestre, T. Carriot, G. Néliat et al., "PK 11195, an antagonist of peripheral type benzodiazepine receptors, modulates BAY K8644 sensitive but not $\beta$ - or $\mathrm{H} 2$-receptor sensitive voltage operated calcium channels in the guinea pig heart," Life Sciences, vol. 39, no. 4, pp. 329-339, 1986. 


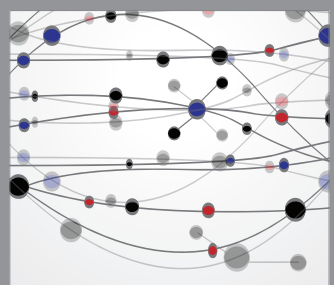

The Scientific World Journal
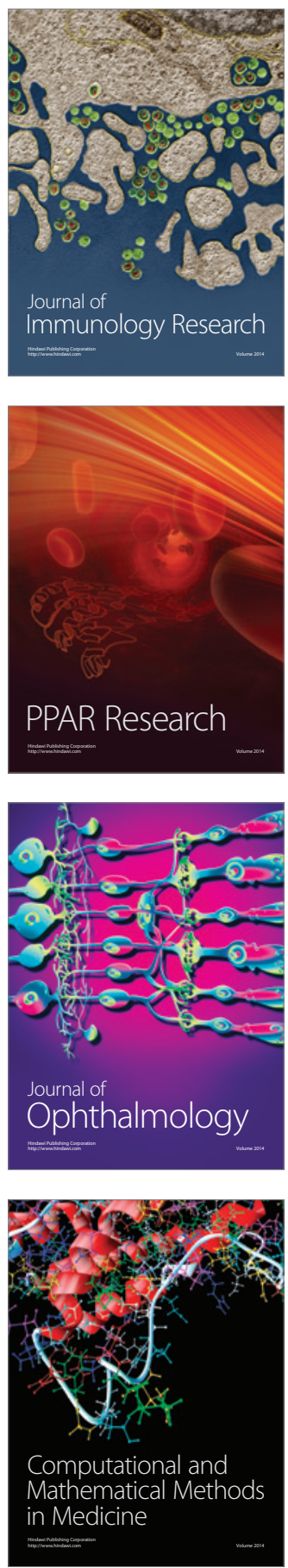

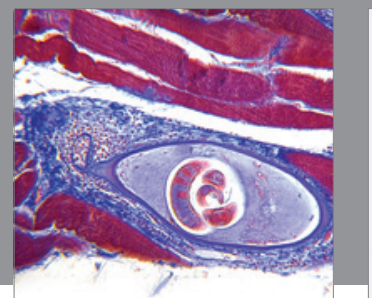

Gastroenterology

Research and Practice
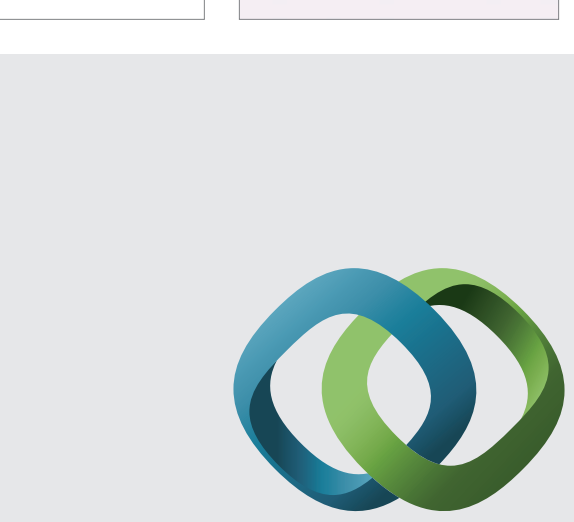

\section{Hindawi}

Submit your manuscripts at

http://www.hindawi.com
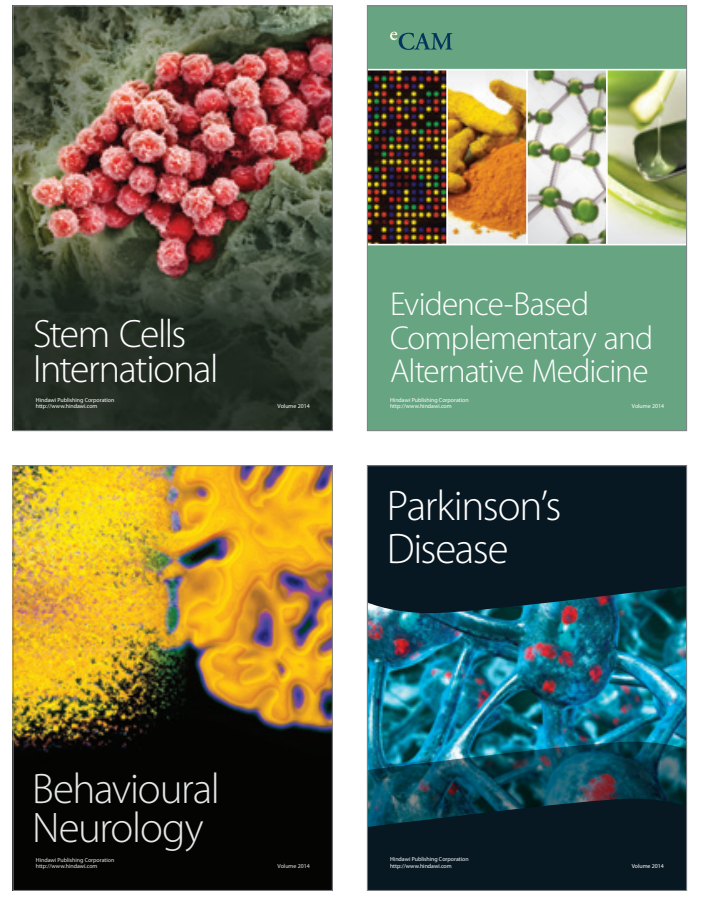
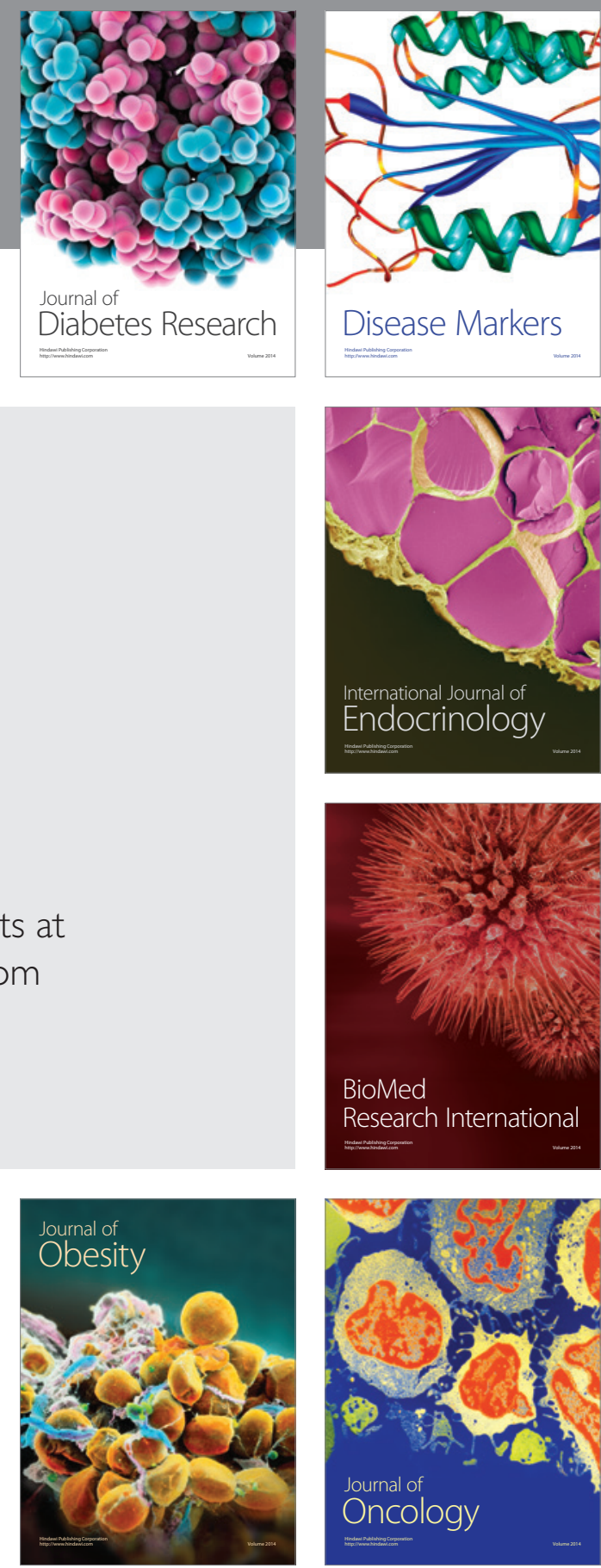

Disease Markers
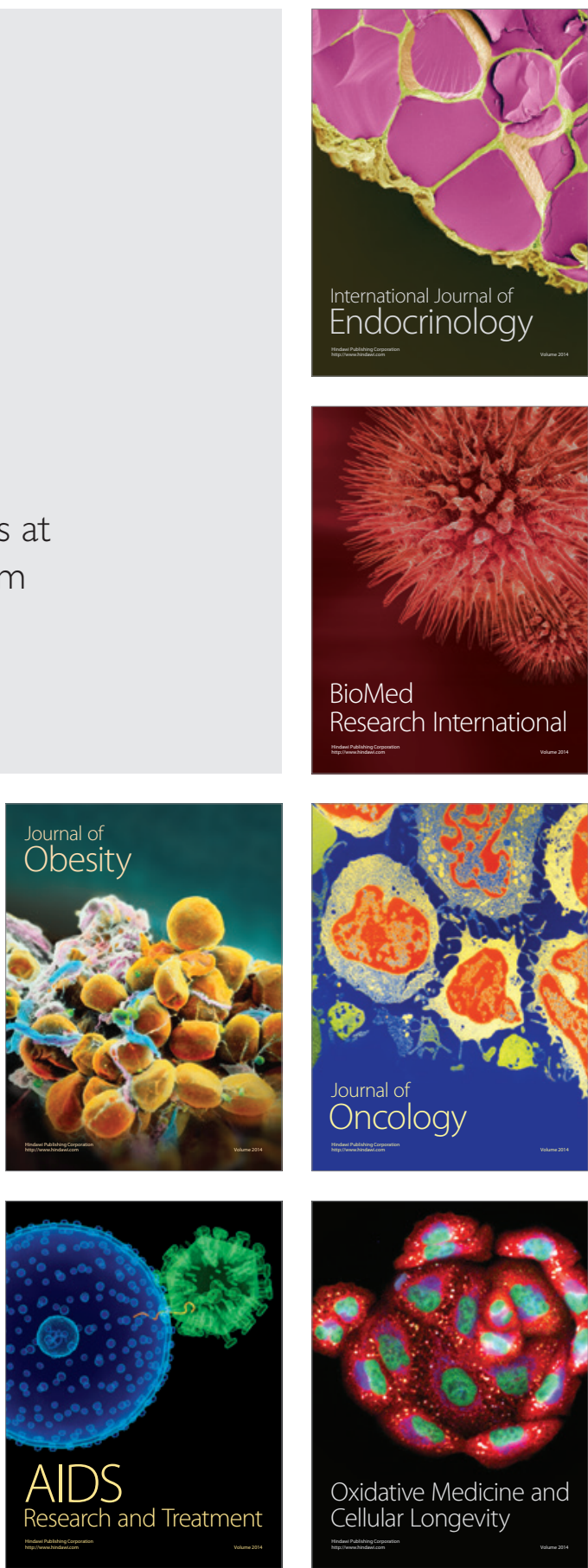